\title{
Quantitative flow ratio en infarto de miocardio para la evaluación de lesiones en arterias no culpables. Estudio piloto QIMERA
}

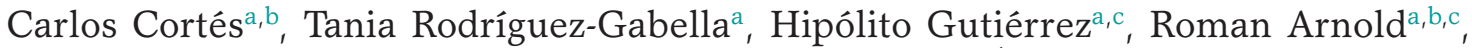 \\ Ana María Serrador ${ }^{a}, b$, Benigno Ramos ${ }^{a}$, Pablo Cataláa, Álvaro Aparisia ${ }^{a}$, Williams Hinojosa ${ }^{a}$, \\ Itziar Gómez ${ }^{a}$, Manuel Carrasco Moralejaa ${ }^{a}$ Juan Luis Gutiérrez-Chico d, José A. San Román ${ }^{a, c}$ \\ e Ignacio J. Amat-Santos ${ }^{a, b}, c, *$ \\ a Departamento de Cardiología, Hospital Clínico Universitario, Valladolid, España \\ b Servicio de Cardiología, Instituto de Ciencias del Corazón, ICICORELAB, Hospital Clínico Universitario, Valladolid, España \\ c Departamento de Cardiología, Hospital Clínico Universitario, Centro de Investigación Biomédica en Red de Enfermedades Cardiovasculares (CIBERCV), \\ Valladolid, España \\ d Departamento de Cardiología, Hospital Punta de Europa, Algeciras, Cádiz, España
}

\section{RESUMEN}

Introducción y objetivos: En pacientes con infarto agudo de miocardio con elevación del segmento ST y enfermedad multivaso se recomienda la revascularización completa. La evaluación funcional con reserva fraccional de flujo (RFF) de las arterias no culpables del infarto es limitada. El quantitative flow ratio (QFR) es una herramienta basada en la angiografía para valorar la gravedad funcional de las lesiones. Se analizaron la evolución funcional de las arterias no culpables del infarto tras la fase aguda y la correlación QFR/RFF en este contexto.

Métodos: Se incluyeron pacientes ingresados con infarto agudo de miocardio con elevación del segmento ST entre enero de 2016 y diciembre de 2017, con enfermedad multivaso y revascularización diferida de lesiones no culpables. Se evaluaron retrospectivamente con QFR durante el procedimiento índice y el diferido, y se evaluó la concordancia QFR/RFF de manera prospectiva entre enero y mayo de 2018.

Resultados: Se incluyeron 131 lesiones de 88 pacientes. Durante el procedimiento índice, el 93,1\% de las lesiones se consideraron significativas de acuerdo con la angiografía, pero solo el 56,3\% cuando se evaluaron con QFR (p < 0,001). El QFR del procedimiento diferido demostró una mayor reducción, con solo el $32,1 \%$ de las lesiones significativas. Todos los pacientes con QFR $\geq 0,82$ durante el procedimiento índice continuaron con estenosis no significativas en la evaluación diferida. La comparación del QFR y la RFF en este contexto demostró buen acuerdo, con una diferencia media de $0,015 \pm 0,02$ ( $p>0,1)$.

Conclusiones: La evaluación fisiológica mediante QFR de las lesiones en arterias no culpables del infarto descartó la significación en el $40 \%$ de las consideradas significativas por angiografía. El valor de QFR se incrementó significativamente del procedimiento índice al diferido, lo que sugiere que en pacientes con QFR $\geq 0,82$ en la fase aguda podrían evitarse procedimientos diferidos innecesarios.

Palabras clave: Reserva fraccional de flujo. Quantitative flow ratio. Arteria no culpable. IAMCEST.

\section{Quantitative flow ratio in myocardial infarction for the evaluation of non-infarct-related arteries. The QIMERA pilot study}

\section{ABSTRACT}

Introduction and objectives: Complete revascularization is recommended for the management of ST-segment elevation myocardial infarctions (STEMI). Although physiological evaluation is recommended for the assessment of nonculprit lesions, in this context, the use of fractional flow reserve (FFR) is limited. The quantitative flow ratio (OFR) is a new angiography-based tool for the assessment of functional severity. We evaluated the functional changes occurring in nonculprit lesions after the acute phase and the QFR/FFR correlation in non-infarct-related arteries.

Methods: We recruited all patients with multivessel disease admitted to our institution due to STEMI from January 2016 through December 2017 who underwent staged interventions for the management of nonculprit lesions. We conducted a retrospective QFR assessment at both the index and the staged procedures and drew a comparison. Also, the QFR/FFR concordance and agreement were prospectively evaluated between January and May 2018 in a cohort of patients with STEMI and multivessel disease. Results: We analyzed a total of 131 lesions in 88 patients. During the initial procedure, $93.1 \%$ of the lesions were considered significant based on the angiography compared to only $56.3 \%$ studied through QFR $(P \leq .001)$. The QFR reassessment during the staged intervention brought this percentage down to $32.1 \%$. All patients with QFR values $\geq 0.82$ during the index procedure

* Autor para correspondencia: Instituto de Ciencias del Corazón, Hospital Clínico Universitario, Ramón y Cajal 3, 47005 Valladolid, España.

Correo electrónico: ijamat@gmail.com (I.J. Amat-Santos).

Recibido el 2 de noviembre de 2018. Aceptado el 18 de diciembre de 2018. Online: 29-04-2019.

Full English text available from: www.recintervcardiol.org/en.

https://doi.org/10.24875/RECIC.M19000017

2604-7306 / @ 2018 Sociedad Española de Cardiología. Publicado por Permanyer Publications. Este es un artículo open access bajo la licencia CC BY-NC-ND 4.0. 
remained nonsignificant at the staged assessment. Both the FFR and the QFR were compared in 12 patients showing good agreement and a mean difference of $0.015 \pm 0.02(P>.1)$.

Conclusions: The QFR-based physiological assessment of nonculprit lesions in STEMI patients led us to consider nonsignificant $40 \%$ of the lesions classified as significant by the angiography. Also, the QFR significantly increased from the acute phase to the staged procedure, indicative that in patients with QFR $\geq 0.82$ in the acute phase a new coronary angiography procedure may be unnecessary.

Keywords: Fractional flow reserve. Quantitative flow ratio. Non-infarct-related artery. STEMI.

Abreviaturas

RFF: reserva fraccional de flujo. ARI: arteria responsable del infarto. ICP: intervención coronaria percutánea. QFR: reserva de flujo cuantitativo. IAMCEST: infarto agudo de miocardio con elevación del segmento ST.

\section{INTRODUCCIÓN}

Hasta el $50 \%$ de los pacientes hospitalizados con cuadros de infarto agudo de miocardio con elevación del segmento ST (IAMCEST) padecen enfermedad multivaso ${ }^{1}$. Hoy en día, se recomienda una revascularización completa antes de proceder al alta hospitalaria, si bien los beneficios derivados de revascularizar la arteria no responsable del infarto (no-ARI) durante una intervención coronaria percutánea (ICP) subsiguiente intervenciones sigue siendo un asunto controvertido ${ }^{1}$. De hecho, la estrategia para el tratamiento de la no-ARI varía enormemente entre los más importantes estudios publicados ${ }^{2-4}$.

La falta de consenso se explica, en parte, por lo imprecisa que es la angiografía a la hora de valorar la gravedad de la estenosis en la noARI, siendo menos del $30-50 \%$ de las lesiones consideradas, inicialmente, aptas para revascularización, confirmadas finalmente mediante reserva fraccional de flujo $(\mathrm{RFF})^{3-4}$. En particular, la gravedad de la estenosis no-ARI suele sobrevalorarse durante la realización de una ICP primaria debido a las condiciones hemodinámicas ${ }^{5}$. No obstante, también en este contexto, las mediciones de la RFF suelen no ser válidas debido a un tono micro y macrovascular alterado o a la obstrucción del flujo microvascular ${ }^{3,6}$. Hay, también, poca literatura sobre la variabilidad de los resultados RFF en lesiones no culpables del infarto. Todos estos factores, probablemente, expliquen por qué la mayoría de los cardiólogos intervencionistas siguen utilizando la angiografía como única herramienta para decir si tratar, o no, una lesión no culpable ${ }^{7}$. La reserva de flujo cuantitativo (QFR, quantitative flow ratio) es una nueva herramienta para valorar la gravedad de las estenosis coronarias que se basa en la dinámica de fluidos computacional y en la reconstrucción tridimensional de la angiografía coronaria sin guía ni necesidad de inducir hiperemia, lo cual guarda una correlación favorable con la RFF en la enfermedad coronaria esta$\mathrm{ble}^{8-10}$. Por otro lado, y aunque se ha sugerido que existe una buena concordancia entre RFF y QFR durante la fase aguda de los infartos de miocardio ${ }^{11}$ es bien sabido que la presencia de disfunción microvascular está asociada a una peor correlación ${ }^{12}$.

Realizamos un estudio piloto para llevar a cabo valoraciones fisiológicas de la gravedad de lesiones no-ARI según QFR durante la realización de ICP primarias y en angiografías diferidas. La correlación existente entre RFF y QFR también se estudió en este contexto.

\section{MÉTODOS}

\section{Diseño del estudio}

Estudio observacional y restrospectivo de un único centro realizado de acuerdo con lo dispuesto en la Declaración de Helsinki y tras obtener la aprobación del comité de ética del hospital. Todos los pacientes incluidos en esta investigación dieron su consentimiento informado para el uso anónimo de sus datos clínicos y de imágenes y solo con fines científicos.

\section{Población del estudio}

El estudio incluyó pacientes consecutivos de edad $\geq 18$ años ingresados en nuestro hospital tras sufrir un IAMCEST entre enero de 2016 y diciembre de 2017 con estenosis coronarias con diámetros $>50 \%$ en arterias no causantes tras valoración angiográfica. Se optó por realizar, en estos pacientes, una estrategia de dos procedimientos a fin de lograr la revascularización completa durante el procedimiento índice. El procedimiento diferido para el tratamiento de las lesiones no causantes se realizó antes del alta hospitalaria tal y como manda el tratamiento estándar en estos casos y siguiendo las recomendaciones actuales ${ }^{1}$. Se incluyó prospectivamente en el estudio a pacientes consecutivos consecutivos de edad $\geq 18$ años ingresados en nuestro hospital tras sufrir un IAMCEST entre enero de 2018 y mayo de 2018 con estenosis coronarias tras valoración angiográfica coronaria en lesiones no-ARI a fin de valorar la concordancia existente entre RFF y QFR. La decisión de realizar valoraciones RFF en estos pacientes se tomó a discreción del operador y en base a criterios intervencionistas y de índole clínica. El nivel de concordancia entre RFF y QFR se estableció en este subgrupo.

Los criterios de exclusión fueron la imposibilidad de dar consentimientos informados, la falta de imágenes angiográficas coronarias válidas para el análisis QFR, la presencia de arterias coronarias normales durante el procedimiento índice, la revascularización quirúrgica y la muerte o la presencia de otras patologías que descartaran revascularización durante el procedimiento índice o contraindicaran el procedimiento diferido durante el mismo ingreso hospitalario.

\section{Valoraciones angiográficas y fisiológicas}

Las proyecciones angiogáficas estandarizadas se realizaron en ambos procedimientos siguiendo el protocolo de adquisición del hospital. La computación de QFR se llevó a cabo offline usando un software específico (QAngio XA 3D prototype, Medis Medical Imaging System, Leiden, Países Bajos). Los detalles de una valoración QFR ya se han descrito con anterioridad en otros estu$\operatorname{dios}^{13}$. En resumen, se utilizaron dos proyecciones con $>25^{\circ}$ de diferencia la una de la otra grabadas a 15 cuadros por segundo para la reconstrucción tridimensional de la no-ARI diana. Se calcularon el diámetro de la estenosis, el área estenótica, el área 
luminal mínima y los diámetros del vaso mínimo y de referencia. Los valores QFR se obtuvieron aplicando principios computacionales de dinámica de fluidos al mencionado software. Se implementó un modelo virtual de la velocidad del flujo hiperémico derivada del flujo de contraste (QFR de contraste, QFRc) sin adenosina. Dos técnicos independientes certificados en software y ciegos a la valoración visual de la gravedad de la estenosis y a los valores QFR obtenidos durante el procedimiento índice, respectivamente, realizaron la valoración offline en un laboratorio central $^{14}$. Se analizó la variabilidad inter e intraobservador en 20 lesiones mediante mediciones repetidas llevadas a cabo por los citados técnicos (10 lesiones a fin de determinar la variabilidad intraobservador y 10 lesiones para valorar la variabilidad inter-observador).

Por último, la correlación entre los valores RFF y QFR se calculó en el grupo prospectivo de pacientes durante los procedimientos índice o diferido. Las mediciones de los valores RFF se llevaron a cabo utilizando el dispositivo Aeris (St. Jude Medical, St. Paul, $\mathrm{MN}$, Estados Unidos). Se indujo hiperemia máxima mediante la infusión continua intravenosa de adenosina $(140-\mu \mathrm{g} / \mathrm{kg} / \mathrm{min})$ que se mantuvo durante dos minutos o hasta la aparición de síntomas. El análisis QFR se llevó a cabo de forma ciega con respecto a los valores $R F F$. Los valores $\leq 0,8$ se consideraron estenosis significativas en la valoración RFF y QFR.

\section{Análisis estadístico}

Las variables cualitativas se expresaron en frecuencias absolutas y porcentajes. Las cuantitativas en medias \pm desviaciones estándar. La distribución normal de las variables cuantitativas se determinó mediante la prueba de Kolmogorov-Smirnov y usando gráficos C-C. Los datos se analizaron paciente por paciente para las características clínicas y vaso por vaso para la angiografía coronaria cuantitativa y para los valores QFR. La concordancia RFF/QFR se determinó usando el método de Bland-Altman y el coeficiente de correlación interclase. Se usó la prueba t para muestras relacionadas para de determinar la evolución de las mediciones tomadas entre el procedimiento índice y el diferido. La curva receiver operating characteristic (ROC) se analizó para valorar la capacidad de la QFR en el procedimiento índice y predecir la QFR en el diferido. Por último, la variabilidad intra e interobservador se determinó utilizando el coeficiente de correlación interclase para estas mediciones repitiéndose el análisis con un mes de diferencia en el $20 \%$ de las lesiones. Todos los análisis se llevaron a cabo usando el paquete estadístico SPSS, versión 24.0 (Armonk, NY: IBM Corpl y R 3.4.3.

\section{RESULTADOS}

\section{Población del estudio}

Ingresaron o fueron derivados a nuestra unidad un total de 828 pacientes con sospecha de IAMCEST entre enero de 2016 y diciembre de 2017. El diagnóstico se confirmó en 455 pacientes, de los cuales $196(43,1 \%)$ revelaron enfermedad multivaso. De estos, 31 pacientes $(15,8 \%)$ fueron sometidos a una revascularización completa durante el procedimiento índice y en 165 pacientes $(84,2 \%)$ la operación de las lesiones no culpables del infarto se pospuso o nunca llegó a realizarse. Por último, se excluyó a 46 pacientes con enfermedad multivaso y un procedimiento diferido por presentar imágenes angiográficas subóptimas que impedían realizar un adecuado análisis QFR durante el procedimiento primario (13 pacientes) o el diferido (33 pacientes). La población del estudio incluyó a 88 pacientes con un total de 131 lesiones en arterias no causantes. Las principales características de la población general pueden consultarse en la tabla 1. La mayoría de los pacientes eran varones $(86,4 \%)$ con IAMCEST inferior o anterior en el $57,9 \%$ y $37,5 \%$, respectivamente, y hospitalizados debido a una ICP primaria en el $64,8 \%$ de los casos. El flujo de grado TIMI de la arteria causante fue $0-1$ en el $51,1 \%$ y el $4,5 \%$ entró en shock. La arteria descendente anterior izquierda fue la no-ARI más frecuente (50\%). Tras el procedimiento índice, la revascularización diferida de $1(56,8 \%)$, $2(38,7 \%)$ o más arterias $(4,5 \%)$ se realizó durante la estancia hospitalaria en unos 5 días de media (rango intercuartílico, 2-6). La revascularización de la ARI y la revascularización completa tuvieron éxito en el $100 \%$ y $76 \%$ de los pacientes, respectivamente.

Tabla 1. Características basales de pacientes hospitalizados tras infarto agudo de miocardio con elevación del segmento ST

\begin{tabular}{|c|c|c|}
\hline Variables & $\begin{array}{l}\begin{array}{l}\text { Muestra } \\
\text { retrospectiva } \\
N=88\end{array} \\
\end{array}$ & $\begin{array}{l}\begin{array}{l}\text { Muestra } \\
\text { prospectiva }\end{array} \\
\mathrm{N}=12\end{array}$ \\
\hline \multicolumn{3}{|l|}{ Características basales } \\
\hline Edad (años) & $67,8 \pm 11,2$ & $70,1 \pm 9,3$ \\
\hline Género (varón) (\%) & 86,4 & 75 \\
\hline Hipertensión (\%) & 45,5 & 66,7 \\
\hline Dislipemia (\%) & 36,4 & 33,3 \\
\hline Altura $(\mathrm{cm})$ & $167,8 \pm 8,2$ & $164,9 \pm 7,8$ \\
\hline Peso (kg) & $79,5 \pm 10,6$ & $78,3 \pm 8,9$ \\
\hline Obesidad $(\%)$ & 29,5 & 33,3 \\
\hline Enfermedad renal crónica $(\%)$ & 3,4 & 0 \\
\hline \multicolumn{3}{|l|}{ Principales características IAMCEST } \\
\hline IAM Killip I (\%) & 85,2 & 100,0 \\
\hline IAM Killip II (\%) & 10,4 & 0 \\
\hline IAM Killip III (\%) & 0 & 0 \\
\hline IAM Killip IV (\%) & 4,5 & 0 \\
\hline \multicolumn{3}{|l|}{ Características de la intervención } \\
\hline Abordaje radial $(\%)$ & 95,3 & 83,3 \\
\hline ICP primaria $(\%)$ & 64,8 & 100,0 \\
\hline ICP rutinaria postrombólisis (\%) & 25 & 0 \\
\hline ICP de rescate $(\%)$ & 10,2 & 0 \\
\hline ARI ADAI (\%) & 40,9 & 50,0 \\
\hline ARI ACD (\%) & 47,8 & 16,7 \\
\hline ARI Cx $(\%)$ & 11,3 & 33,3 \\
\hline Flujo de grado 0 TIMI (\%) & 44,3 & 41,7 \\
\hline Flujo de grado 1 TIMI (\%) & 6,8 & 25,0 \\
\hline Flujo de grado 2 TIMI (\%) & 3,4 & 0,0 \\
\hline Flujo de grado 3 TIMI (\%) & 45,5 & 33,3 \\
\hline $\begin{array}{l}\text { Tiempo hasta procedimiento } \\
\text { diferido (días) }\end{array}$ & $5,8 \pm 3,6$ & N/A \\
\hline
\end{tabular}

Los datos se expresan en $n(\%)$ o media \pm desviación estándar. ACD: arteria coronaria derecha; ADAI: arteria descendente anterior izquierda; ARI: arteria responsable del infarto; $\mathrm{Cx}$ : arteria circunfleja; IAM: infarto agudo de miocardio; IAMCEST: infarto agudo de miocardio con elevación del segmento ST; ICP: intervención coronaria percutánea; TIMI: Thrombolysis in Myocardial Infarction. 


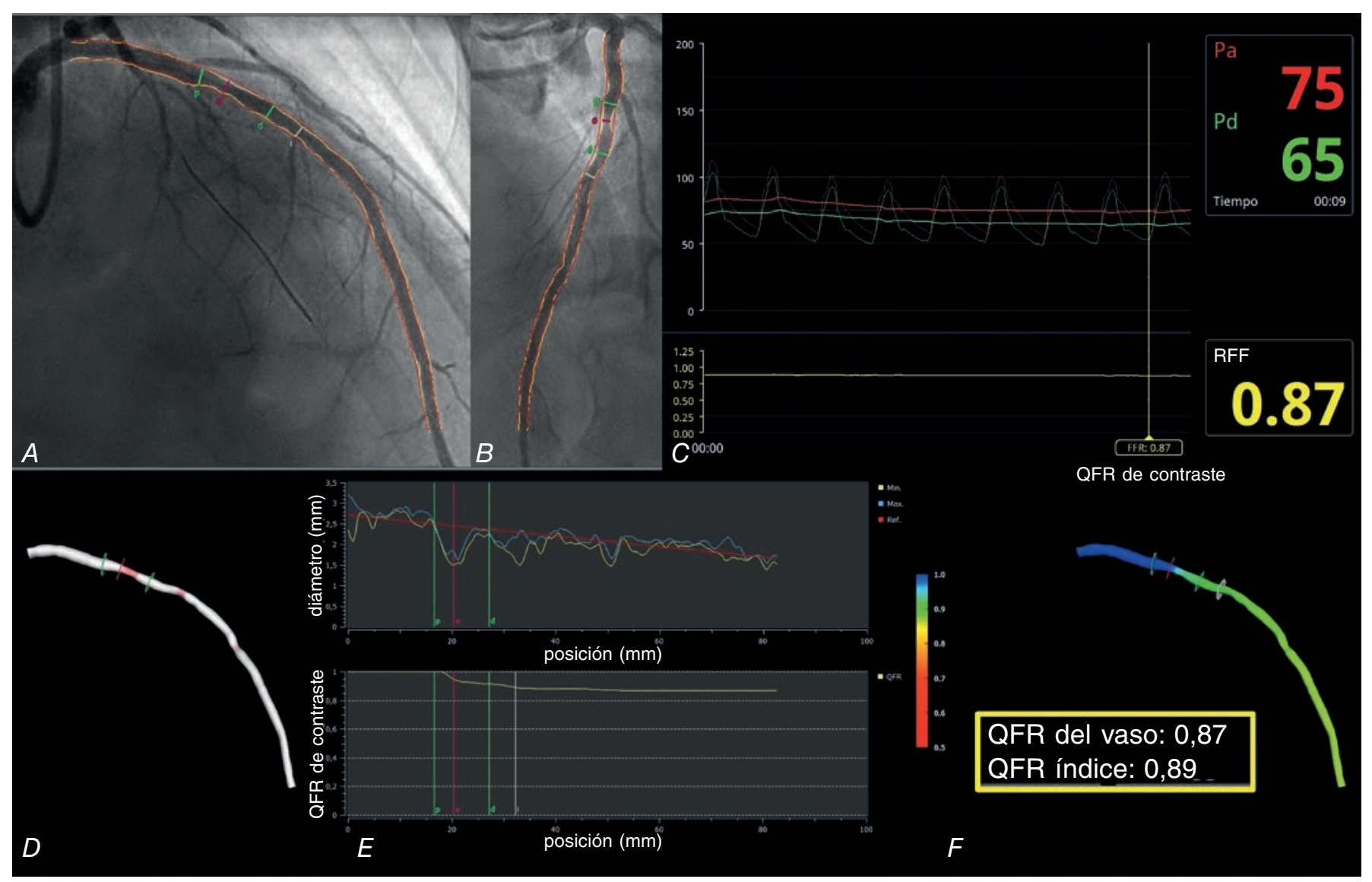

Figura 1. Ejemplo representativo de concordancia entre RFF y QFR en lesión en no-ARI. A: OAD, $30^{\circ}$; $\mathrm{CRA}, 30^{\circ}$; B: OAI, 40ㅜ CRA, $20^{\circ}$; C: RFF; D: reconstrucción angiográfia en 3D. E: diámetro luminal y retirada QFR; F: valores QFR. ARI: arteria no responsable del infarto; CRA: craneal; OAD: oblicua anterior derecha; OAI: oblicua anterior izquierda; QFR: ratio de flujo cuantitativo; RFF: reserva fraccional de flujo.

\section{Validación de la valoración QFR en lesiones no culpables}

La valoración prospectiva de la correlación entre QFR y RFF se llevó a cabo en 12 pacientes (15 lesiones) de la población del estudio siguiendo el protocolo ya descrito en estudios publicados ${ }^{11}$. Las principales características de esta muestra de validación fueron similares a las de la cohorte QFR tal y como muestra la tabla 1. No hubo ninguna complicación tras el uso de las guías de presión. Los valores RFF medios fueron $0,87 \pm 0,06$ y la diferencia media con respecto a los valores QFR, 0,017 $\pm 0,02$. La figura 1 muestra un ejemplo paradigmático de una lesión evaluada mediante $\mathrm{RFF}$ y QFR. El coeficiente de correlación interclase fue 0,959 (intervalo de confianza del 95\%, 0,882-0,986). En 4 lesiones $(26,7 \%)$ el análisis de la RFF se llevó a cabo durante el procedimiento índice tras la revascularización de la lesión causante. En esta muestra no se observó diferencia alguna en la precisión diagnóstica de la QFR entre el procedimiento índice y el diferido (diferencia media relativa $0,0346 \pm 0,29$ y $0,114 \pm 0,10, p=0,214$ ); se utilizó el método de Bland-Altman para ver el grado de concordancia entre estas mediciones y la correlación según el procedimiento descrito en la figura 2 .

\section{Cambios QFR a lo largo de los procedimientos índice y diferido}

Ciento veintidós $(93,1 \%)$ de las 131 lesiones en vasos no causantes se consideraron aptas para ser sometidas a revascularizaciones percutáneas programadas según valoración visual de la angiografía. De estas, solo el $56,3 \%$ arrojaron valores QFR $\leq 0,80$ en el procedimiento índice cuando se valoraron retrospectivamente (figura 3). Se pudo confirmar una caída sin relevancia estadística de los valores QFR entre el procedimiento índice y el diferido en pacientes con valores QFR iniciales > 0,80; no obstante, 2 pacientes con valores QFR inicialmente irrelevantes experimentaron una caída $<0,80$ en la valoración de la angiografía diferida. Todos los pacientes con valores iniciales $>0,82$ confirmaron la presencia de estenosis en el segundo procedimiento. Por otro lado, el $45,9 \%$ de las lesiones con valores QFR significativos fueron consideradas irrelevantes cuando fueron valoradas durante el segundo procedimiento, con diámetros medios y áreas estenóticas más grandes ( $p<0,001$ para ambos) tal y como revela la tabla 2. Los principales cambios observados entre ambos procedimientos se muestran en la figura 4 y en el ejemplo paradigmático de la figura 5. La sensibilidad y especificidad de valores QFR $>0,82$ durante el procedimiento índice para predecir estenosis importantes $(\mathrm{QFR}<0,80$ ) durante el procedimiento programado fueron del 84 y $58,7 \%$, respectivamente, con un valor predictivo positivo del $52,5 \%$ y un valor predictivo negativo del $87 \%$ (figura 6). La estrategia terapéutica se implementó con independencia de los hallazgos de la valoración de QFR ya que se hizo una estimación de ésta retrospectivamente. Esto nos permitió comparar la estrategia en base a la interpretación de la valoración de la angiografía visual y posteriores hallazgos QFR en un total de 46 lesiones tratadas con stents a pesar de presentar valores QFR irrelevantes. 

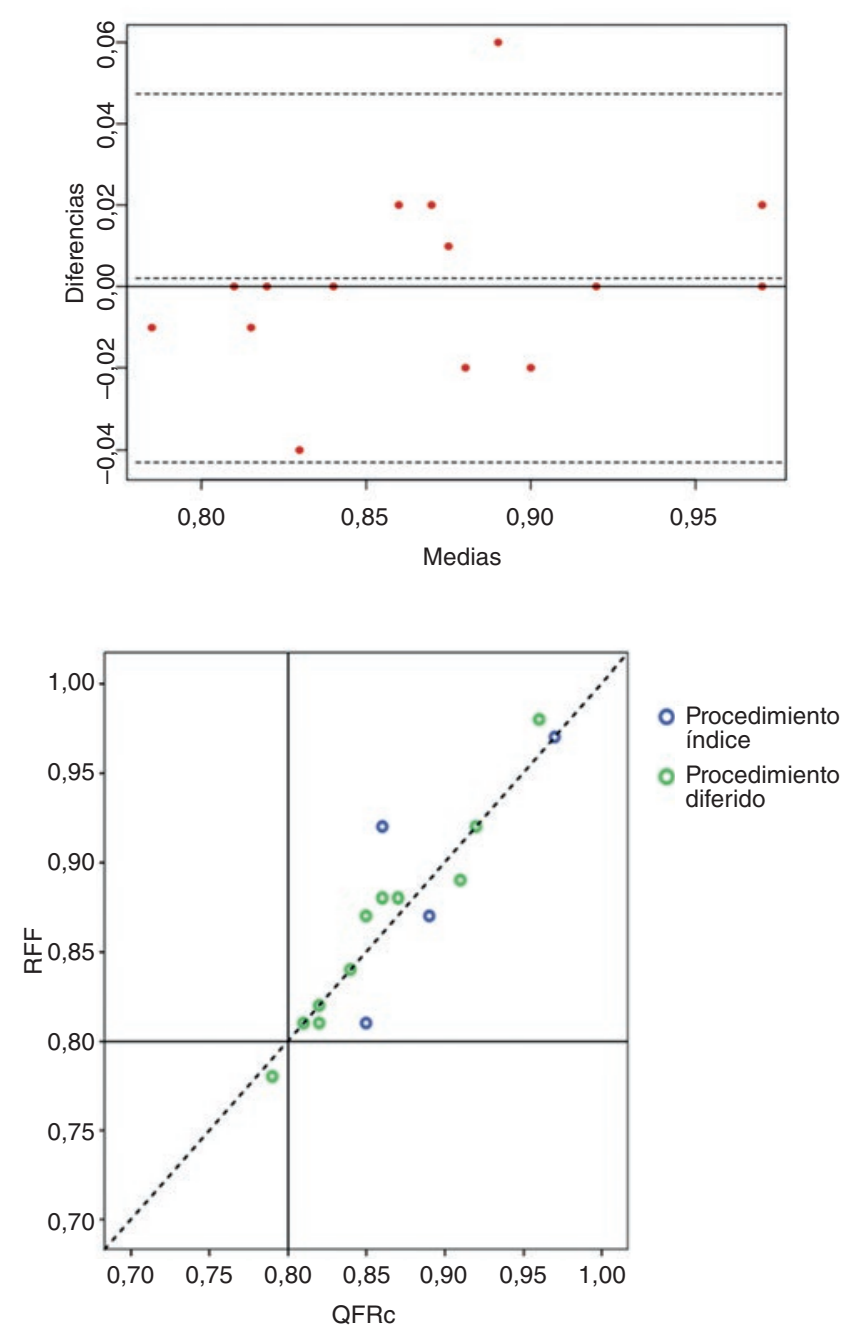

Figura 2. Correlación y concordancia entre RFF y QFR en lesión en estenosis en la arteria no responsable del infarto basada en el procedimiento índice o diferido. QFR: ratio de flujo cuantitativo; RFF: reserva fraccional de flujo.

\section{Variabilidad intra e interobservador}

La variabilidad intra e interobservador para calcular los valores QFR se confirmó a través de los coeficientes de correlación interclase de 0,958 (intervalo de confianza del 95\%, 0,877-0,984) y 0,991 (intervalo de confianza del 95\%, 0,960-0,997), respectivamente.

\section{DISCUSIÓN}

Es bien sabido que la valoración visual de la estenosis coronaria suele sobrevalorar su gravedad de esta ${ }^{5}$, aún así, los operadores son reacios a llevar a cabo valoraciones funcionales de lesiones no culpables en el contexto del IAMCEST debido tanto a los posibles riesgos asociados a la RFF como a la fisiología alterada de esta patología. Los valores QFR para la valoración de lesiones no causantes en pacientes que han sufrido un IAMCEST ya se ha investigado en un pequeño estudio piloto ${ }^{8}$ y ofrece posibles ventajas, basadas principalmente, en su rápida aplicación y en que no es preciso pasar una guía por las arterias coronarias ni administrar adenosina. Los principales hallazgos de nuestro estudio son: a) la
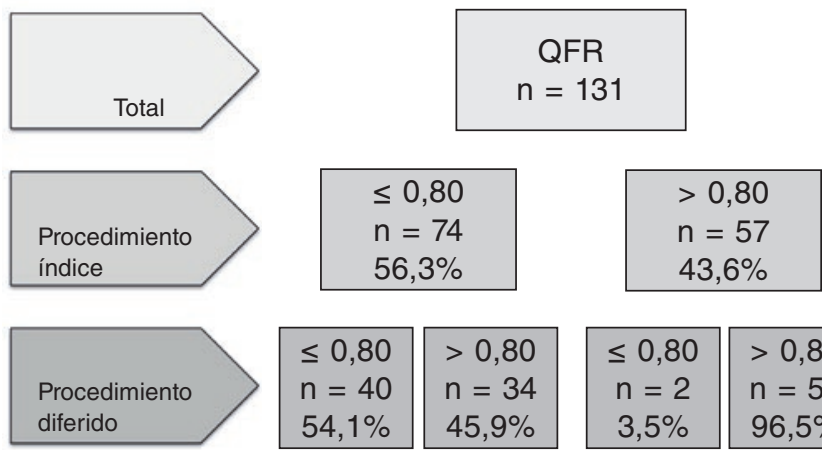

\begin{tabular}{|l|l|}
\hline$\leq 0,80$ & $>0,80$ \\
$n=40$ & $n=34$ \\
$54,1 \%$ & $45,9 \%$ \\
\hline
\end{tabular}

\begin{tabular}{c|c}
$\leq 0,80$ & $>0,80$ \\
$n=2$ & $n=55$ \\
$3,5 \%$ & $96,5 \%$
\end{tabular}

Figura 3. Cambios QFR en los procedimientos índice y diferido en pacientes con estenosis significativa confirmada mediante angiografía en vasos no culpables. QFR: ratio de flujo cuantitativo.

Tabla 2. Mediciones mediante angiografía coronaria cuantitativa y análisis de la ratio de flujo cuantitativo según el procedimiento.

\begin{tabular}{|c|c|c|c|}
\hline Total: 131 no-ARI & $\begin{array}{l}\text { Procedi- } \\
\text { miento } \\
\text { índice }\end{array}$ & $\begin{array}{l}\text { Segundo } \\
\text { procedi- } \\
\text { miento }\end{array}$ & $\mathbf{p}^{*}$ \\
\hline $\begin{array}{l}\text { Diámetro de estenosis } \\
(\%)\end{array}$ & $58,9 \pm 12,0$ & $51,15 \pm 10,6$ & $<0,001$ \\
\hline Área estenótica (\%) & $70,1 \pm 15,1$ & $63,9 \pm 15,1$ & $<0,001$ \\
\hline $\begin{array}{l}\text { Diámetro máximo del } \\
\text { vaso proximal }(\mathrm{mm})\end{array}$ & $2,7 \pm 0,6$ & $2,8 \pm 0,6$ & 0,182 \\
\hline $\begin{array}{l}\text { Diámetro mínimo del } \\
\text { vaso proximal }(\mathrm{mm})\end{array}$ & $2,4 \pm 0,5$ & $2,5 \pm 0,6$ & 0,231 \\
\hline $\begin{array}{l}\text { Diámetro máximo del } \\
\text { vaso distal }(\mathrm{mm})\end{array}$ & $2,6 \pm 0,7$ & $2,6 \pm 0,6$ & 0,850 \\
\hline $\begin{array}{l}\text { Diámetro mínimo del } \\
\text { vaso distal }(\mathrm{mm})\end{array}$ & $2,3 \pm 0,6$ & $2,3 \pm 0,5$ & 0,751 \\
\hline $\begin{array}{l}\text { Diámetro del vaso de } \\
\text { referencia }(\mathrm{mm})\end{array}$ & $2,5 \pm 0,7$ & $2,5 \pm 0,6$ & 0,295 \\
\hline $\begin{array}{l}\text { Diámetro luminal } \\
\text { mínimo }(\mathrm{mm})\end{array}$ & $1,0 \pm 0,3$ & $1,2 \pm 0,4$ & $<0,001$ \\
\hline $\begin{array}{l}\text { Ratio de flujo } \\
\text { cuantitativo }\end{array}$ & $0,76 \pm 0,14$ & $0,82 \pm 0,12$ & $<0,001$ \\
\hline
\end{tabular}

ARI: arteria responsable del infarto.

*Valores p significativos en negrita.

QFR tiene una buena concordancia con la RFF y una variabilidad intra e interobservador óptima, en operadores experimentados en la valoración de la gravedad funcional de lesiones coronarias, lo cual sugiere que podría ser una herramienta excelente para el manejo de cuadros de IAMCEST; b) la gravedad de la estenosis en lesiones no culpables es más alta durante la fase aguda del IAMCEST, lo cual, en gran medida, nos confirma la angiografía pero también la QFR; c) la valoración funcional de las lesiones no culpables mediante QFR podría ser útil durante la fase aguda del IAMCEST. Por otro lado, las estenosis con valores QFR > 0,82 siguieron siendo irrelevantes durante el seguimiento en todos los casos, lo cual evitaría tener que realizar procedimientos diferidos e implantar stents hasta en la tercera parte de estos pacientes; además, unos valores QFR significativos durante el procedimiento índice no deberían llevar a tratar la lesión durante la misma intervención ya que, según la QFR, el 45,9\% de las lesiones significativas dejaron de serlo durante la valoración diferida. 


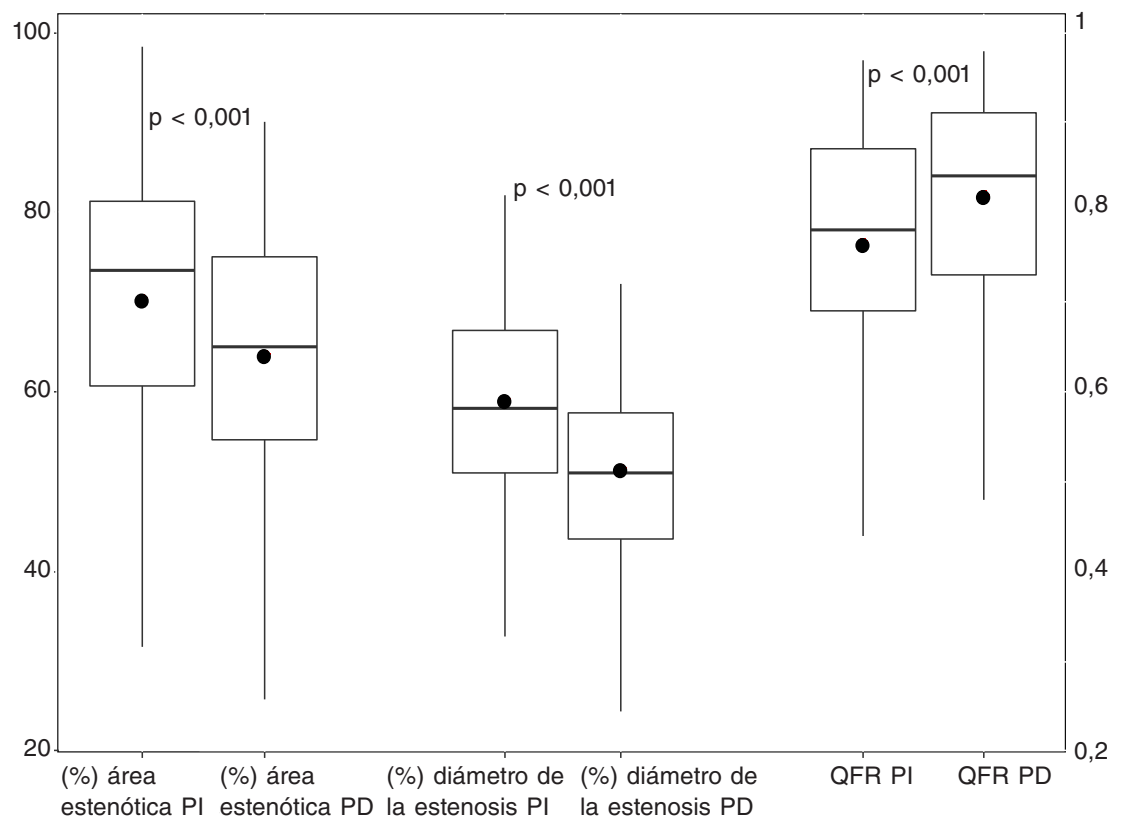

- Representa la media

Figura 4. Cambios QFR en lesiones no culpables en los procedimientos índice y diferido en pacientes con estenosis significativa confirmada mediante angiografía. PD: procedimiento diferido; PI: procedimiento índice; QFR: ratio de flujo cuantitativo.

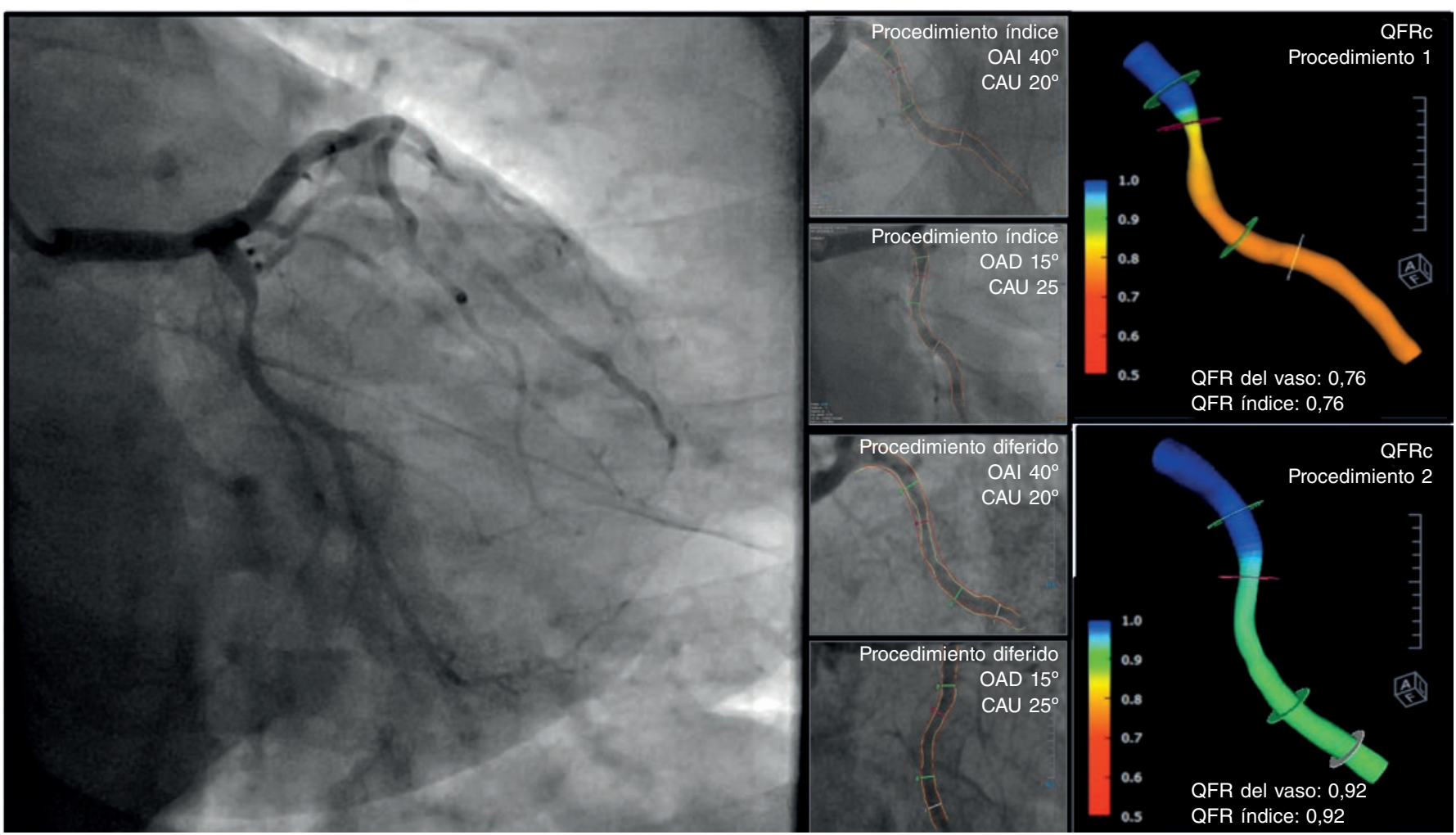

Figura 5. Cambios en los valores QFR para la arteria circunfleja durante los procedimientos índice y diferido en paciente con IAMCEST inferior secundario a una oclusión en la coronaria derecha. IAMCEST: infarto agudo de miocardio con elevación del segmento ST; QFR: ratio de flujo cuantitativo.

\section{Diferencias en las valoraciones anatómicas y fisiológicas}

La cada vez mayor evidencia que sitúa la fisiología por encima de la anatomía en el campo de la enfermedad coronaria merece un análisis específico en pacientes que sufren un IAMCEST a fin de intentar reducir los índices de sobretratamiento. Ya se han descrito en este contexto sobrevaloraciones cuando se toman decisiones basadas en angiografías ${ }^{5}$ e infravaloraciones cuando se toman basadas en la RFF ${ }^{6}$. En el estudio COMPARE-ACUTE, la valoración fisiológica de no-ARI se realizó durante el procedimiento índice y reveló que solo la mitad de las lesiones consideradas importantes en la angiografía se confirmaron posteriormente en 


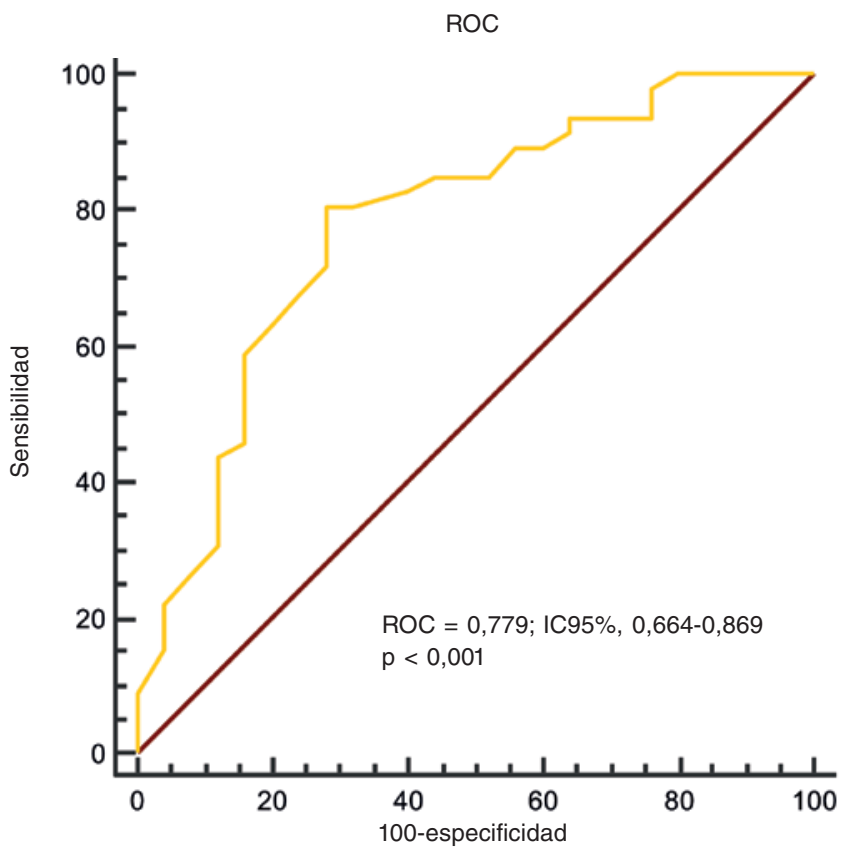

Figura 6. El análisis de la curva ROC revela la sensibilidad y especificidad de QFR > 0,82 durante el procedimiento índice para predecir estenosis significativa durante el procedimiento diferido según repetidas mediciones QFR. IC95\%: intervalo de confianza del 95\%; QFR: ratio de flujo cuantitativo; ROC: característica operativa del receptor.

la $\mathrm{RFF}^{3}$. Y al contrario, en el estudio DANAMI-3-PRIMULTI, la valoración de lesiones no culpables se realizó durante procedimientos diferidos, lo cual elevó el porcentaje de lesiones con valores $\mathrm{RFF}<0,80$ hasta casi el $70 \%{ }^{4}$. Las alteraciones del tono macrovascular o la obstrucción del flujo microvascular durante la fase aguda de los infartos de miocardio explicaría en parte estos hallazgos de estudios más extensos. Esta diferencia a la hora de estimar la gravedad entre ambos procedimientos mediante angiografías coronarias cuantitativas y QFR debería tenerse en cuenta para evitar tratar lesiones en no-ARI durante la realización de ICP primarias. Algo importantísimo aun cuando solo se tenga en cuenta la valoración angiográfica, ya que la necesidad de realizar revascularizaciones completas sigue siendo objeto de debate y es algo que todavía no recomiendan las guías de práctica clínica actuales.

\section{Posibles nuevas aportaciones de la QFR en IAMCEST}

La QFR puede obtenerse de manera segura durante la realización de una intervención coronaria percutánea primaria. Un punto de corte de 0,82 ayuda a identificar a aquellos pacientes no candidatos a revascularización secuencial. La valoración de la QFR durante el procedimiento diferido no reveló diferencias significativas frente a la fase aguda, probablemente debido al limitado tamaño de la muestra. No obstante, sí se observó una cierta tendencia hacia valores QFR más altos en el procedimiento diferido, de forma similar a la RFF. La presencia de disfunción microvascular durante la fase aguda podría explicarlo ${ }^{12}$, aunque calidad de la angiografía coronaria también podría influir. En cualquier caso, el potencial de la QFR en no-ARI para identificar lesiones que no deberían tratarse y así evitar procedimientos diferidos innecesarios es sumamente interesante. Según esta perspectiva, la QFR podría ser esa tan anhelada herramienta que nos ayudase a determinar cuál es la mejor estrategia a la hora de tomar la difícil decisión de tratar la enfermedad multivaso en pacientes con IAMCEST. Los estudios actualmente en marcha que están poniendo a prueba esta hipótesis y valorando la necesidad de realizar revascularizaciones urgentes durante el seguimiento con esta nueva estrategia determinarán la relevancia clínica de la QFR.

\section{Limitaciones}

La principal limitación de este estudio es su naturaleza retrospectiva y el limitado tamaño de la muestra. La valoración QFR precisa de la realización de estudios angiográficos de calidad y, aunque se elaboró un protocolo estándar, de forma rutinaria, para las angiografías coronarias, varios estudios tuvieron que ser excluidos por no tener la calidad adecuada, lo cual podría ser un sesgo que deberían analizar futuros estudios. Además, el pequeño tamaño de la muestra podría tener que ver con el limitado potencial de precisión diagnóstica de la QFR en ciertos subgrupos de pacientes y con la propia validación de la QFR frente a la RFF.

\section{CONCLUSIONES}

En resumen, las valoraciones funcionales coronarias basadas en la QFR de lesiones no culpables tras infarto agudo de miocardio arrojaron un porcentaje alto de sobrevaloración angiográfica en la gravedad de las estenosis (> 40\%). Valores QFR $>0,82$ durante el procedimiento índice identifican de manera precisa las lesiones no culpables y valores por debajo de este umbral sugieren la necesidad de nuevas valoraciones fisiológicas antes de a recomendar la angioplastia. La validación prospectiva de esta hipótesis está plenamente justificada.

\section{FINANCIACIÓN}

Este proyecto, con el código GRS1728/A/18, ha sido financiado por la Gerencia Regional de Salud de la Junta de Castilla y León de España.

\section{CONFLICTOS DE INTERESES}

No se declara ninguno.

\section{¿QUÉ SE SABE DEL TEMA?}

- Más de la mitad de los pacientes hospitalizados por IAMCEST presentan enfermedad multivaso, razón por la cual se recomienda una revascularización completa.

- La valoración funcional de las lesiones no culpables del infarto tras un IAMCEST ha resultado ser útil a la hora de establecer la estrategia de revascularización a seguir; sin embargo, la mayoría de los cardiólogos intervencionistas basan su decisión en la angiografía por los retos y limitaciones que presenta la RFF en este contexto.

- La QFR es un nuevo índice funcional basado en la reconstrucción tridimensional de la anatomía coronaria y en la dinámica de fluidos computacional que guarda una estrecha relación con la RFF y no acarrea la necesidad de usar guías en las arterias coronarias. 


\section{¿QUÉ APORTA DE NUEVO?}

- Un buen grado de concordancia entre la QFR y la RFF confirmado para las lesiones no culpables en pacientes sufren un IAMCEST.

- Los valores QFR durante la fase aguda de un IAMCEST sugirieron una mayor gravedad que las valoraciones diferidas. Umbrales QFR $=0,82$ en la fase aguda identificaron mejor al umbral de pacientes que podrían no precisar procedimientos diferidos por nuevas valoraciones funcionales o angioplastias en las lesiones no causantes, lo cual, a su vez, minimizaría riesgos y costes innecesarios.

\section{BIBLIOGRAFÍA}

1. Ibanez B, James S, Agewall S, et al. 2017 ESC Guidelines for the management of acute myocardial infarction in patients presenting with ST-segment elevation: The Task Force for the management of acute myocardial infarction in patients presenting with ST-segment elevation of the European Society of Cardiology (ESC). Eur Heart J. 2018;39:119-177.

2. Gershlick AH, Khan JN, Kelly DJ, et al. Randomized trial of complete versus lesion-only revascularization in patients undergoing primary percutaneous coronary intervention for STEMI and multivessel disease: The CvLPRIT trial. J Am Coll Cardiol. 2015;65:963-972.

3. Smits PC, Abdel-Wahab M, Neumann FJ, et al. Fractional Flow ReserveGuided Multivessel Angioplasty in Myocardial Infarction. $N$ Engl J Med. 2017;376:1234-1244.

4. Engstrøm T, Kelbæk H, Helqvist $\mathrm{S}$, et al. Complete revascularisation versus treatment of the culprit lesion only in patients with ST-segment elevation myocardial infarction and multivessel disease (DANAMI-3-PRIMULTI): an open-label, randomised controlled trial. Lancet. 2015;386:665-671.

5. Hanratty CG, Koyama Y, Rasmussen HH, et al. Exaggeration of Nonculprit Stenosis Severity During Acute Myocardial Infarction: Implications for Imme- diate Multivessel Revascularization. J Am Coll Cardiol. 2002;40:911 916

6. Cuculi F, Maria L de, Meier $\mathrm{P}$, et al. Impact of Microvascular Obstruction on the Assessment of Coronary Flow Reserve, Index of Microcirculatory Resistance, and Fractional Flow Reserve After ST-Segment Elevation Myocardial Infarction. J Am Coll Cardiol. 2014;64:1894-1904.

7. Toth GG, Toth $\mathrm{B}$, Johnson NP, et al. Revascularization decisions in patients with stable angina and intermediate lesions: Results of the international survey on interventional strategy. Circ Cardiovasc Interv. 2014;7:751759 .

8. Tu S, Westra J, Yang J, et al. Diagnostic Accuracy of Fast Computational Approaches to Derive Fractional Flow Reserve From Diagnostic Coronary Angiography. JACC Cardiovasc Interv. 2016;9:2024-2035.

9. Yazaki K, Otsuka M, Kataoka S, et al. Applicability of 3-Dimensional Quantitative Coronary Angiography-Derived Computed Fractional Flow Reserve for Intermediate Coronary Stenosis. Circ J. 2017;81:988-992.

10. Westra J, Tu S, Nissen L, et al. Evaluation of Coronary Artery Stenosis by Quantitative Flow Ratio During Invasive Coronary Angiography. Circ Cardiovasc Imaing. 2018;11:1-8.

11. Spitaleri G, Tebaldi M, Biscaglia S, et al. Quantitative Flow Ratio Identifies Nonculprit Coronary Lesions Requiring Revascularization in Patients With ST-Segment-Elevation Myocardial Infarction and Multivessel Disease. Circ Cardiovasc Interv. 2018;11:e006023.

12. Mejía-Rentería H, Lee JM, Lauri F, et al. Influence of Microcirculatory Dysfunction on Angiography-Based Functional Assessment of Coronary Stenoses. JACC Cardiovasc Interv. 2018;11:741-753.

13. Tu S, Barbato E, Yang J, Li Y, Rusinaru D, Reiber JHC. Fractional Flow Reserve Calculation From 3-Dimensional Quantitative Coronary Angiography and TIMI Frame Count. JACC Cardiovasc Interv. 2014;7:768-777.

14. Instituto de Ciencias del Corazón. Disponible en: http://icicorelab.es. Consultado 14 Jul 2018. 